CERN-TH/2002-341

NEIP-02-010

CPTH-RR-083-1102

hep-ph/0211409

\title{
Brane to bulk supersymmetry breaking and radion force at micron distances
}

\author{
I. Antoniadis ${ }^{1 *}$ K. Benakli ${ }^{1,2} \dagger$, A. Laugier ${ }^{1,3}$, T. Maillard ${ }^{1,4}$ \\ ${ }^{1}$ CERN Theory Division CH-1211, Genève 23, Switzerland \\ ${ }^{2}$ Institut de Physique, Université de Neuchâtel, \\ CH-2000 Neuchâtel, Switzerland \\ ${ }^{3}$ Centre de Physique Théorique, Ecole Polytechnique, \\ 91128 Palaiseau, France \\ ${ }^{4}$ Institut für Theoretische Physik, ETH Hönggerberg \\ CH-8093 Zürich, Switzerland
}

\begin{abstract}
We study mediation of supersymmetry breaking in the bulk, in models with primordial supersymmetry breaking on D-branes at the string scale, in the $\mathrm{TeV}$ region. We compute the gravitino and scalar masses up to one-loop level, as well as the radion coupling to matter. We find that the latter mediates a model independent force at submillimeter distances that can be tested in micro-gravity experiments for any dimensionality of the bulk. In the case of two large dimensions, our type I string framework provides an example which allows to stabilize the radion potential and determine the desired hierarchy between the string and Planck scales.
\end{abstract}

\footnotetext{
* On leave of absence from CPHT, Ecole Polytechnique, UMR du CNRS 7644.

†Permanent address: LPTHE, Universités de Paris VI et VII, UMR du CNRS 7589.
} 


\section{Introduction}

An interesting class of type I string models with large internal dimensions [1, 2] is when the closed string bulk is supersymmetric, while supersymmetry is broken on the world volume of a particular brane configuration [3, 4. This framework guarantees the absence of quadratic divergences in the cosmological constant which is of order $M_{s}^{4}$ with $M_{s}$ the string scale, without larger contributions proportional to $M_{s}^{2} M_{P}^{2}$ with $M_{P}$ the Planck mass. On the other hand, it offers two distinct possibilities for realizing the Standard Model. (i) It can be localized on a non supersymmetric brane configuration, in which case the string scale must be in the $\mathrm{TeV}$ region to protect the gauge hierarchy [2]. (ii) It can be located on some supersymmetric branes at a different position, in which case the string scale should be at intermediate energies [5], since mediation of supersymmetry breaking in the observable world will be suppressed by the size of the bulk.

In both cases above, one important question is to understand the mediation of supersymmetry breaking in the bulk. In this work, we address this question and we compute the gravitino and bulk scalar masses, up to one loop level. Our framework is a class of type I string models, where supersymmetry breaking is due to combinations of D-branes and orientifolds which preserve different amount of supersymmetry. The particle spectrum on these D-branes is then non-supersymmetric, but supersymmetry is realized nonlinearly on their worldvolume which contains a (tree-level) massless goldstino 6. 4. These models have in general a localized tree-level potential, arising at the disk worldsheet, and thus non-vanishing tadpoles of NS-NS (NeveuSchwarz) scalar fields. In order to avoid this problem, we can introduce a tiny source of supersymmetry breaking in the bulk, using Scherk-Schwarz boundary conditions along the lines of Ref. 4], that vanishes in the decompactification limit. However, as it will be clear later, our results for scalar masses do not depend on this modification, since the one-loop mediation of supersymmetry breaking comes entirely from the branes.

We find that for more than two large bulk dimensions, the scalar masses are always lighter, of the order of $M_{s}^{2} / M_{P}$, as expected by the effective field theory, while the gravitino (and other closed string fermions) is in general much heavier, of the order of the compactification scale $1 / R$. This is because fermions acquire tree-level masses from the Scherk-Schwarz boundary conditions, while proper loop corrections from the brane are extremely suppressed. The two scales coincide in the case where the bulk is two-dimensional (2d), since $M_{s}^{2} / M_{P} \sim 1 / R^{n / 2}$ for $n$ bulk dimensions. Also, in the special case of $n=1$, both masses become proportional to $1 / R$.

An immediate consequence of our results is that in models with the string 
scale in the $\mathrm{TeV}$ region, the radion, the universal scalar modulus whose vacuum expectation value (VEV) determines the size of the bulk, mediates a new force at submillimeter distances. We thus compute its coupling to matter and find that it is comparable to gravity and depends only on the number of extra dimensions. Therefore, this force can be tested in tabletop experiments that test gravity at very short distances [7, independently of the dimensionality of the bulk. This is in contrast to the modification of the Newton's law which is testable only when there are two large dimensions.

Another important question is vacuum stability. Although the general issue goes beyond the scope of this paper, we address the problem of stabilizing the radion [8], assuming that the dilaton VEV is fixed and thus the string coupling. For this purpose, we study in particular the case of $n=2$ bulk dimensions, where there are logarithmic corrections depending on the size of the bulk $\left[9 .{ }^{1}\right.$ We then compute the effective potential and show that the compactification scale can be fixed at values which are hierarchically different than the string scale, providing the desired hierarchy between $M_{s}$ and $M_{P}$.

Our paper is organized as follows. In Section 2, we derive from the effective field theory the radion mass and its coupling to matter, using the general form of the scalar potential that we compute in the following sections. In Section 3, we describe our framework and we construct a class of models that generalize those of ref. 4] and we use for our subsequent one-loop calculations. In Section 4, we compute the one-loop effective potential in the case of two bulk dimensions and we fix the radion VEV. In Section 5, we compute the string one-loop corrections to the bulk scalar masses and to the gravitino mass. Finally, in Section 6, we present our summary and discuss concluding remarks.

\section{Radion mass and couplings}

The kinetic terms of the radion can be obtained upon dimensional reduction of the higher dimensional bulk Einstein-Hilbert action:

$$
S_{\text {bulk }}^{(4+n)}=\frac{M_{s}^{2+n}(2 \pi)^{1-n}}{g_{s}^{2}} \int d^{4+n} x \sqrt{G} \mathcal{R}^{(4+n)},
$$

where we consider a bulk with $n$ large extra dimensions, while the remaining $6-n$ are compactified at the string scale. $\mathrm{G}$ is the corresponding metric and $\mathcal{R}^{(4+n)}$ is the Ricci scalar. Here, we assumed that the dilaton VEV is

\footnotetext{
${ }^{1}$ For a recent analysis in the $n=1$ dimensional case, see ref. [10].
} 
fixed and thus the string coupling $g_{s}$ is constant. Parametrizing the metric $G=\operatorname{diag}\left(g, R^{2} \mathbf{1}\right)$, with $g$ the four-dimensional (4d) metric and $R$ a common compactification radius, one finds in the string frame:

$$
S_{\text {kin }}^{(4)}=\frac{2 \pi M_{s}^{2+n}}{g_{s}^{2}} \int d^{4} x \sqrt{g} R^{n}\left\{\mathcal{R}^{(4)}-n(n-1)\left(\frac{\partial R}{R}\right)^{2}\right\} .
$$

Letting now $R_{0}$ the $\mathrm{VEV}$ of $R$ and defining the radion field $\varphi$ by

$$
R=R_{0} e^{\kappa \varphi} \quad ; \quad M_{P}=\frac{2^{5 / 2} \pi M_{s}^{(2+n) / 2}}{g_{s}} R_{0}^{n / 2} \quad ; \quad \frac{1}{2 \kappa^{2}}=\frac{M_{P}^{2}}{16 \pi}
$$

the action (2.2) becomes:

$$
S_{\text {kin }}^{(4)}=\int d^{4} x e^{n \kappa \varphi}\left[\frac{1}{2 \kappa^{2}} \mathcal{R}^{(4)}-\frac{n(n-1)}{2}(\partial \varphi)^{2}\right] .
$$

The loop corrections induce a potential localized on the brane, where supersymmetry is broken:

$$
S_{\mathrm{pot}}^{(4)}=\int d^{4} x \Lambda(R)=M_{s}^{4} \int d^{4} x\left\{\frac{1}{g_{s}} V_{0}+V_{1}(R)+\mathcal{O}\left(g_{s}\right)\right\},
$$

where $V_{0}$ is a constant contribution originating from the disk worldsheet and $V_{1}(R)$ is the one-loop correction that we compute in Section 4. In models without NS-NS tadpoles, that we present in the next section, the tree-level potential $V_{0}$ vanishes, while $V_{1}(R)$ behaves in the large radius limit as:

$$
R \rightarrow \infty: \quad V_{1}(R) \sim \begin{cases}\text { constant } & \text { for } n>2 \\ \ln R & \text { for } n=2 \\ R & \text { for } n=1\end{cases}
$$

The constant behavior follows from the localization of the potential, while the logarithmic and linear in $R$ corrections are characteristic in the cases where the bulk is two- and one-dimensional, respectively, due to the corresponding infrared growth of the propagation of massless fields 9 .

One can now go to the Einstein frame by rescaling the four-dimensional (4d) metric $g_{\mu \nu} \rightarrow g_{\mu \nu} e^{-n \kappa \varphi}$, which also diagonalizes the radion and graviton kinetic terms:

$$
S_{\mathrm{tot}}^{(4)}=\int d^{4} x\left[\frac{1}{2 \kappa^{2}} \mathcal{R}^{(4)}+\frac{n(n+2)}{4}(\partial \varphi)^{2}+e^{-2 n \kappa \varphi} \Lambda(R)\right] .
$$

Assuming that the full potential has a minimum at $R=R_{0}$, and using eqs. (2.5) and (2.6), it follows that in the large volume limit the radion mass 
is of order $M_{s}^{2} / M_{P}$ for any number of dimensions $n>1$. The case of $n=1$ is special. There is an apparent enhancement factor $\sqrt{R_{0} M_{s}}$, but as we will see in Section 4 the mixing among Kaluza-Klein (KK) modes becomes important and after taking it into account, one finds a value proportional to the compactification scale $1 / R$. Thus, for $n>1$ and for a string scale $M_{s} \simeq 1-10$ $\mathrm{TeV}$, the radion mass is of the order of $10^{-4}-10^{-6} \mathrm{eV}$, which corresponds to a wavelength of the order of 1 millimeter to 10 microns. Note that in the case of two-dimensional $(2 \mathrm{~d})$ bulk, there is an enhancement factor of the radion mass by $\ln R_{0} M_{s} \simeq 30$ which decreases its wavelength by roughly an order of magnitude.

The coupling of the radion to matter can be deduced easily from the rescaling of the metric which changes the string to the Einstein frame, and the normalization of its kinetic term in the action (2.7). Considering two masses at rest, $m_{1}$ and $m_{2}$, the amplitudes corresponding to the exchange of a graviton and of a radion of momentum $p$ are:

$$
\frac{2 \kappa^{2} m_{1} m_{2}}{p^{2}} \quad \text { and } \quad \frac{n}{n+2} \frac{2 \kappa^{2} m_{1} m_{2}}{p^{2}},
$$

respectively. It follows that the radion couples universally as gravity, with an attractive force of relative strength $\alpha$ :

$$
\alpha=\frac{n}{n+2}
$$

depending only on the dimensionality of the bulk $n$ [1]. The values of this coupling vary from $\alpha=1 / 2$ to $\alpha=3 / 4$ for $n=2$ to $n=6$ large extra dimensions. Moreover, in the case of $n=2$, there may be again model dependent logarithmic corrections of the order of $\left(g_{s} / 4 \pi\right) \ln R_{0} M_{s} \simeq$ $\mathcal{O}(1)$. Such a force can be tested in microgravity experiments and should be contrasted with the change of Newton's law due the presence of extra dimensions that is observable only for $n=2$ [7]. In Fig. 1, we plot our predictions together with the present and future experimental limits.

A final remark concerns the masses generated for other massless scalars in the bulk. Since supersymmetry is broken only locally, on the worldvolume of the branes, one can apply the same argument used for the radion. All such scalars $\Phi$ are expected to receive from loop corrections localized mass corrections, so that the relevant $4 \mathrm{~d}$ effective action for the zero-mode reads:

$$
S_{\mathrm{kin}}^{(4)}=\int d^{4} x \frac{1}{2}\left[\left(R_{0} M_{s}\right)^{n}(\partial \Phi)^{2}+c M_{s}^{2} \Phi^{2}\right],
$$

where the kinetic term comes from the bulk, as in eq. (2.2), while the mass term is localized and is fixed by the string scale, up to a numerical constant 


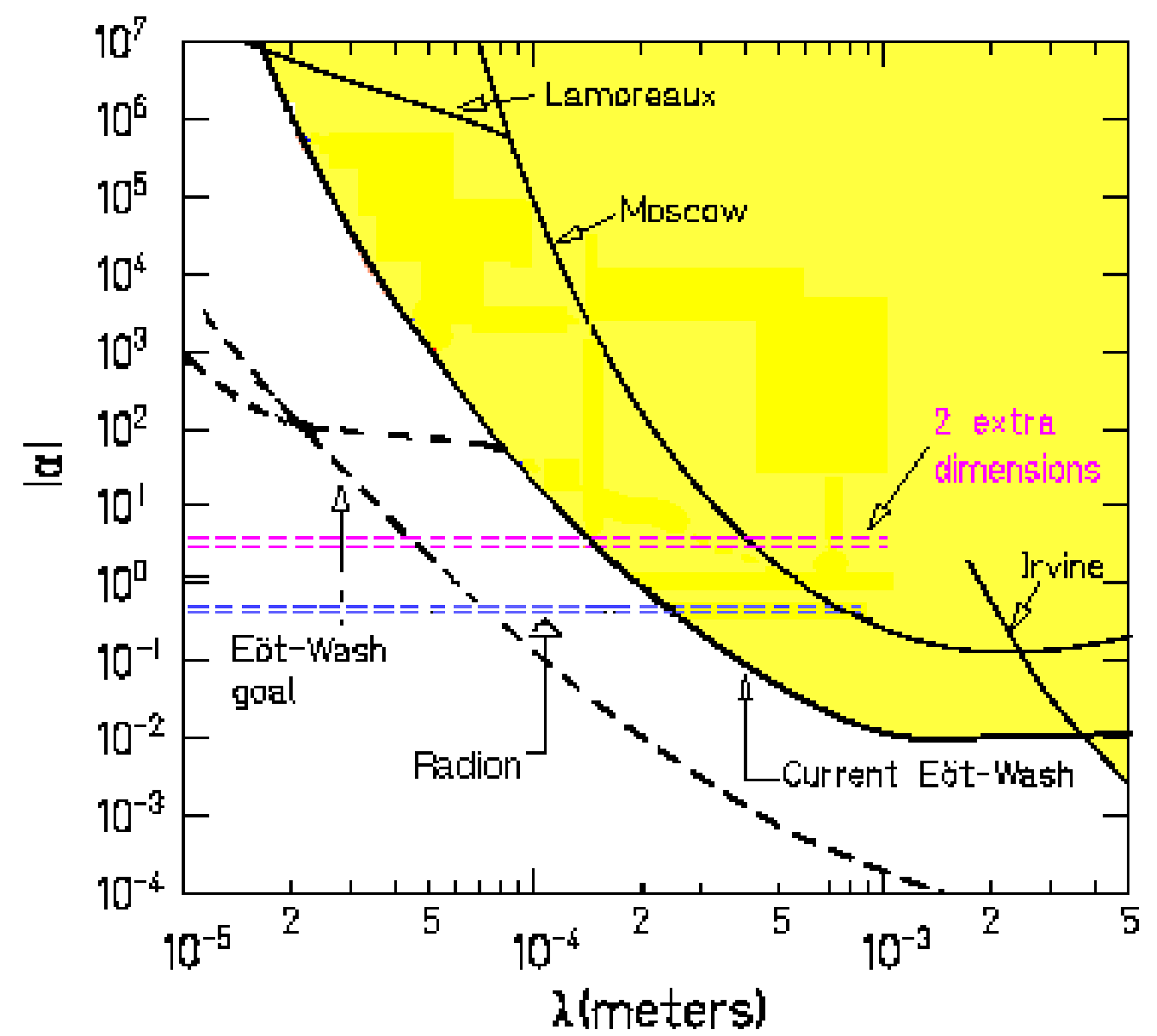

Figure 1: Present limits on non-Newtonian forces at short distances (gray regions), compared to new forces mediated by the graviton in the case of two large extra dimensions, and by the radion.

$c$, in analogy with the radion potential (2.5). It follows that the physical scalar mass is suppressed by the volume of the bulk, and is thus of the order of $M_{s}^{2} / M_{P}$. The couplings of these scalars to matter from our world brane are in general model dependent and may lead to violations of the equivalence principle at short distances that could be measured experimentally.

\section{$3 \quad$ Model building}

To illustrate the discussion of Section 2 we will consider some type IIB string orientifold examples which involve compactification on a six-dimensional space, where $n$ dimensions have a common large compactification radius 


\begin{tabular}{|c||c|c|c|c||c|c|}
\hline \multicolumn{1}{|c||}{} & \multicolumn{4}{c||}{ Orientifolds } & \multicolumn{2}{c|}{ D-branes } \\
\hline \hline Symbol & $\mathrm{O}_{p}^{-}$ & $\overline{\mathrm{O}}_{p}^{-}$ & $\mathrm{O}_{p}^{+}$ & $\overline{\mathrm{O}}_{p}^{+}$ & $\mathrm{D} p$ & $\overline{\mathrm{D}} p$ \\
\hline RR charge & - & + & + & - & + & - \\
\hline NS-NS charge & - & - & + & + & + & + \\
\hline Number & $N_{p}^{-}$ & $\bar{N}_{p}^{-}$ & $N_{p}^{+}$ & $\bar{N}_{p}^{+}$ & $n_{p}$ & $\bar{n}_{p}$ \\
\hline Supersymmetries & $Q$ & $\bar{Q}$ & $Q$ & $\bar{Q}$ & $Q, \bar{Q}_{\mathrm{NL}}$ & $\bar{Q}, Q_{\mathrm{NL}}$ \\
\hline
\end{tabular}

Table 1: The RR and NS-NS charges of orientifolds and D-branes.

$R>>l_{s} \equiv M_{s}^{-1}$, while the remaining $6-n$ have a string scale size and do not play a major role.

The simplest cases on which the desired computations can be carried out consist of toroidal compactifications with the adjunction of a certain number of orientifold planes and D-branes. These come in different kinds (see Table 1) as they are distinguished by the charges they carry, both Neveu-SchwarzNeveu-Schwarz (NS-NS), or equivalently their tension, and Ramond-Ramond (RR) type. Here, we will consider for simplicity D-branes and orientifold planes of the same dimensionality $p$. The generalization to the case of several types extended in different internal dimensions is straightforward.

In our conventions, D-branes (anti-D-branes) have positive (negative) RR charge and positive tension, while there are two types of orientifolds: $\mathrm{O}^{-}-$ planes with negative tension and $\mathrm{O}^{+}$-planes with positive tension. Thus, $\mathrm{O}^{+}$-planes $\left(\overline{\mathrm{O}}^{+}\right.$-planes) have the same quantum numbers as D-branes $(\overline{\mathrm{D}}-$ branes), while $\mathrm{O}^{-}$-planes $\left(\overline{\mathrm{O}}^{-}\right.$-planes) have opposite ones. In Table 1 , we also display the supersymmetries preserved. D-branes and both types of orientifolds preserve the same amount of supersymmetry $Q$, which amounts half of the bulk supersymmetry, while their conjugates $\overline{\mathrm{D}}$ and $\overline{\mathrm{O}}, \overline{\mathrm{O}}^{ \pm}$preserve the other half $\bar{Q}$. On the other hand, D-branes ( $\overline{\mathrm{D}}$-branes) realize also the other half $\bar{Q}(Q)$ in a non-linear way, indicated with a subscript NL in the table.

Let us specialized for definiteness to the case of two transverse dimensions with D7-branes and orientifold 7-planes, $p=7$. The total RR charge of the system:

$$
Q^{R R}=-8 N_{p}^{-}+8 \bar{N}_{p}^{-}+8 N_{p}^{+}-8 \bar{N}_{p}^{+}+n_{p}-\bar{n}_{p}
$$

is constrained to vanish, $Q^{R R}=0$, due to the flux conservation inside the compact space. On the other hand, the total NS-NS charge of the system is 
given by:

$$
Q^{N S}=-8 N_{p}^{-}-8 \bar{N}_{p}^{-}+8 N_{p}^{+}+8 \bar{N}_{p}^{+}+n_{p}+\bar{n}_{p}
$$

is not required to vanish in general by consistency. However, a non-vanishing $Q^{N S}$ implies a non-vanishing tadpole for the corresponding NS-NS scalar (in our case the dilaton), accompanied by a non-trivial (usually runaway) treelevel potential. Following the method of ref. 4], we will start by constructing models with no tadpoles, that correspond to consistent non-supersymmetric string vacua in flat space at the tree-level. Since we are interested to stabilize the compactification radius at values much larger than the string length, we will specialize to the case of a two-dimensional bulk and compute the oneloop radion potential which behaves logarithmically in the large radius limit, according to eq. (2.6) . However, we will be unable to find consistent solutions in this limit.

On the other hand, since the vacuum is non-supersymmetric, higher loop corrections are expected to generate in any case a non-vanishing dilaton potential, and thus dilaton tadpole, related to the familiar problem of the cosmological constant, for which we have nothing new to say. We will therefore allow, in a second step, the presence of a potential, and thus of an uncanceled dilaton tadpole, already at the tree-level. We will then show that one can stabilize the radion and determine the desired hierarchy by combining the tree-level contribution with the logarithmic one-loop corrections, in a way analogous to the no-scale mechanism or the so-called inverse hierarchy of softly broken supersymmetry [12].

As we already stated in the introduction, we will assume that the dilaton VEV is fixed and ignore the presence of a cosmological constant at the minimum of the potential, focusing only on fixing the radion. Finally, the compactification will proceed in two steps: first down to $p+1=10-n$ and then down to four. Here, we will describe in detail only the first step, while the second is trivial for toroidal or orbifold compactifications.

\subsection{Susy breaking along one extra dimension}

In [4, a construction of consistent type I vacua with spontaneously broken supersymmetry was presented. The main ingredients of the construction are the following:

- The ten-dimensional type IIB string is compactified on $X \otimes \mathcal{M}_{4}$ with an orientifold projection on the worldsheet. Here $\mathcal{M}_{4}$ is the fourdimensional Minkowski space and $X$ is a compact manifold which contains a segment $S^{1} / Z_{2}$ used to break supersymmetry in the bulk. 
The breaking is achieved by a choice of different periodicity conditions around $S^{1}$ for the fermions and bosons in the same supermultiplets (Scherck-Schwarz mechanism). In our construction we choose all the bosons periodic, while the fermions are anti-periodic.

- At each of the end-points of the segment $S^{1} / Z_{2}$, the orientifold projection introduces an orientifold plane. The Scherck-Schwarz (SS) boundary conditions require the two orientifolds to preserve different halves of the bulk supersymmetry, which is achieved for instance by having the orientifolds to be of the same type and to carry opposite RR charges.

- In order to insure the absence of NS-NS tadpoles, we require that the orientifolds carry negative NS-NS charges which are cancelled by adding D-branes. The cancellation of the total RR charge on the other hand implies that the D-branes appear in pairs of brane-anti-brane.

- Two possible consistent models are found. In the first case (named "brane supersymmetry") the branes are placed on top of orientifolds preserving the same supersymmetries. The massless modes localized at the boundaries are then degenerate between bosons and fermions and form supermultiplets. A second case (named "brane supersymmetry breaking") is obtained by putting the branes on top of orientifolds preserving different supersymmetries. The massless modes left on the branes are not anymore degenerate between bosons and fermions but the effective field theory has a non-linearly realized supersymmetry. These properties are easily understandable by inspection of Table 1 .

\subsection{Models with two compact dimensions}

In this section, we present an extension of the above construction with branes and anti-branes located at different points of a two-dimensional compactification plane instead of a line. We will consider two cases, where the nonperiodic boundary conditions for bulk fermions are imposed along one or both directions. We will denote the two classes of models as $(I)$ and $(I I)$.

The one-loop partition function contains four terms accounting for the contributions of the torus $(\mathcal{T})$, the Klein bottle $(\mathcal{K})$, the annulus $(\mathcal{A})$ and the Möbius strip $(\mathcal{M})$. For simplicity, we will consider an orthogonal torus with equal radii $R$. Also, below we present only the simplest models defined effectively in eight dimensions. The generalization to lower dimensions, with less supersymmetry in the bulk, and various types of D-branes is straightforward and can be easily done along the lines of refs. 4]. 
The torus contribution is given by (see [4 for the integration measures):

$$
\begin{aligned}
\mathcal{T}_{I}= & {\left[E_{0}^{\prime}\left(V_{8} \bar{V}_{8}+S_{8} \bar{S}_{8}\right)+O_{0}^{\prime}\left(I_{8} \bar{I}_{8}+C_{8} \bar{C}_{8}\right)\right.} \\
& \left.-E_{\frac{1}{2}}^{\prime}\left(V_{8} \bar{S}_{8}+S_{8} \bar{V}_{8}\right)-O_{\frac{1}{2}}^{\prime}\left(I_{8} \bar{C}_{8}+C_{8} \bar{I}_{8}\right)\right] \sum_{m, n} Z_{m, n}
\end{aligned}
$$

in the case where the SS boundary condition is used along one direction, and by:

$$
\begin{aligned}
\mathcal{T}_{I I}= & \left(E_{0}^{\prime} E_{0}^{\prime}+O_{0}^{\prime} O_{0}^{\prime}\right)\left(V_{8} \bar{V}_{8}+S_{8} \bar{S}_{8}\right) \\
& +\left(E_{0}^{\prime} O_{0}^{\prime}+O_{0}^{\prime} E_{0}^{\prime}\right)\left(I_{8} \bar{I}_{8}+C_{8} \bar{C}_{8}\right) \\
& -\left(E_{1 / 2}^{\prime} E_{1 / 2}^{\prime}+O_{1 / 2}^{\prime} O_{1 / 2}^{\prime}\right)\left(V_{8} \bar{S}_{8}+S_{8} \bar{V}_{8}\right) \\
& -\left(E_{1 / 2}^{\prime} O_{1 / 2}^{\prime}+O_{1 / 2}^{\prime} E_{1 / 2}^{\prime}\right)\left(I_{8} \bar{C}_{8}+C_{8} \bar{I}_{8}\right)
\end{aligned}
$$

for the case where the SS deformation is used along both directions. Our notations are the same as in ref. [4]. $I_{8}, V_{8}, S_{8}$ and $C_{8}$ are the $S O(8)$ characters corresponding to the conjugacy classes of the identity, vector, spinor and conjugate spinor, respectively. Their expressions in terms of theta-functions are given by:

$$
I_{8}=\frac{\theta_{3}^{4}+\theta_{4}^{4}}{2 \eta^{4}} \quad V_{8}=\frac{\theta_{3}^{4}-\theta_{4}^{4}}{2 \eta^{4}} \quad S_{8}=\frac{\theta_{2}^{4}+\theta_{1}^{4}}{2 \eta^{4}} \quad C_{8}=\frac{\theta_{2}^{4}-\theta_{1}^{4}}{2 \eta^{4}},
$$

where $\eta$ is the Dedekind eta-function. $Z_{m, n}$ is the one-dimensional torus partition function, while $E_{a}^{\prime}\left(O_{a}^{\prime}\right)$ denotes the partition function restricted to even (odd) windings $n$ and shifted momenta $m+a$ :

$Z_{m, n}=\frac{1}{|\eta|^{2}} q^{\frac{\alpha^{\prime}}{4}\left(\frac{m}{R}+\frac{n R}{\alpha^{\prime}}\right)^{2}} \bar{q}^{\frac{\alpha^{\prime}}{4}\left(\frac{m}{R}-\frac{n R}{\alpha^{\prime}}\right)^{2}} \quad E_{a}^{\prime}=\sum_{m, n} Z_{m+a, 2 n} \quad O_{a}^{\prime}=\sum_{m, n} Z_{m+a, 2 n+1}$

with $\alpha^{\prime}=l_{s}^{2}, q=e^{2 i \pi \tau}$ and $\tau$ the complex modulus of the worldsheet torus.

The Klein bottle contribution can be written as:

$$
\begin{aligned}
\mathcal{K}_{I} & =\frac{1}{2}\left(\left(V_{8}-S_{8}\right) \sum_{m_{1}} \tilde{Z}_{2 m_{1}}+\left(I_{8}-C_{8}\right) \sum_{m_{1}} \tilde{Z}_{2 m_{1}+1}\right) \sum_{m_{2}} \tilde{Z}_{m_{2}} \\
\mathcal{K}_{I I} & =\frac{1}{2}\left(V_{8}-S_{8}\right) \sum_{m_{1}+m_{2} \text { even }} \tilde{Z}_{m_{1}} \tilde{Z}_{m_{2}}+\frac{1}{2}\left(I_{8}-C_{8}\right) \sum_{m_{1}+m_{2} \text { odd }} \tilde{Z}_{m_{1}} \tilde{Z}_{m_{2}}
\end{aligned}
$$

in the direct (open string) channel where

$$
\tilde{Z}_{n}=\frac{q^{\frac{n^{2} R^{2}}{2 \alpha^{\prime}}}}{\eta}
$$


and

$$
\begin{aligned}
\tilde{\mathcal{K}}_{I} & =\frac{2^{5}}{2} \alpha^{\prime} R^{-2}\left(V_{8} \sum_{n_{1}} Z_{2 n_{1}}-S_{8} \sum_{n_{1}} Z_{2 n_{1}+1}\right) \sum_{n_{2}} Z_{2 n_{2}} \\
\tilde{\mathcal{K}}_{I I} & =\frac{2^{5}}{2} \alpha^{\prime} R^{-2}\left(V_{8} Z_{e e}-S_{8} Z_{o o}\right)
\end{aligned}
$$

in the transverse (closed string) channel. Here again we used the notation:

$$
Z_{m}=\frac{q^{\frac{\alpha^{\prime}}{4}\left(\frac{m}{R}\right)^{2}}}{\eta}
$$

while $Z_{e e}$ and $Z_{o o}$ are the two-dimensional partition function sums, restricted to (even, even) and (odd, odd) momenta, respectively.

The remaining two contributions describe the content of the open string sector in these models. The annulus vacuum amplitude, in the transverse channel, can be expressed as:

$$
\begin{aligned}
\tilde{\mathcal{A}}= & \frac{2^{-5}}{2} \frac{\alpha^{\prime}}{R^{2}}\left(\alpha_{1}^{2} V_{8} Z_{e e}+\alpha_{2}^{2} V_{8} Z_{o o}-\beta_{1}^{2} S_{8} Z_{e e}-\beta_{2}^{2} S_{8} Z_{o o}+\gamma_{1}^{2} V_{8} Z_{e o}\right. \\
& \left.+\gamma_{2}^{2} V_{8} Z_{o e}-\delta_{1}^{2} S_{8} Z_{e o}-\delta_{2}^{2} S_{8} Z_{o e}\right)
\end{aligned}
$$

where $\alpha_{j}, \beta_{j}, \gamma_{j}, \delta_{j}(j=1,2)$ are the boundary reflection coefficients. As usual, an appropriate parametrization in terms of the Chan-Paton factors is given by:

$$
\begin{aligned}
\alpha_{1} & =n_{00}+n_{\pi \pi}+\bar{n}_{\pi \pi}+n_{\pi 0}+\bar{n}_{\pi 0}+\bar{n}_{00}+n_{0 \pi}+\bar{n}_{0 \pi} \\
\alpha_{2} & =n_{00}+n_{\pi \pi}+\bar{n}_{\pi \pi}-n_{\pi 0}-\bar{n}_{\pi 0}+\bar{n}_{00}-n_{0 \pi}-\bar{n}_{0 \pi} \\
\beta_{1} & =n_{00}+n_{\pi \pi}-\bar{n}_{\pi \pi}+n_{\pi 0}-\bar{n}_{\pi 0}-\bar{n}_{00}+n_{0 \pi}-\bar{n}_{0 \pi} \\
\beta_{2} & =n_{00}+n_{\pi \pi}-\bar{n}_{\pi \pi}-n_{\pi 0}+\bar{n}_{\pi 0}-\bar{n}_{00}-n_{0 \pi}+\bar{n}_{0 \pi} \\
\gamma_{1} & =n_{00}-n_{\pi \pi}-\bar{n}_{\pi \pi}+n_{\pi 0}+\bar{n}_{\pi 0}+\bar{n}_{00}-n_{0 \pi}-\bar{n}_{0 \pi} \\
\gamma_{2} & =n_{00}-n_{\pi \pi}-\bar{n}_{\pi \pi}-n_{\pi 0}-\bar{n}_{\pi 0}+\bar{n}_{00}+n_{0 \pi}+\bar{n}_{0 \pi} \\
\delta_{1} & =n_{00}-n_{\pi \pi}+\bar{n}_{\pi \pi}+n_{\pi 0}-\bar{n}_{\pi 0}-\bar{n}_{00}-n_{0 \pi}+\bar{n}_{0 \pi} \\
\delta_{2} & =n_{00}-n_{\pi \pi}+\bar{n}_{\pi \pi}-n_{\pi 0}+\bar{n}_{\pi 0}-\bar{n}_{00}+n_{0 \pi}-\bar{n}_{0 \pi}
\end{aligned}
$$

where $n_{y_{1} y_{2}}\left(\bar{n}_{y_{1} y_{2}}\right)$ represents the number of branes (anti-branes) sitting at the point $\left(y_{1} y_{2}\right)$ with $y_{1,2}=0$ or $\pi R$.

With this parametrization, the direct channel annulus amplitude takes 
the form:

$$
\begin{aligned}
\mathcal{A} & =\left(V_{8}-S_{8}\right)\left\{\frac{n_{00}^{2}+\bar{n}_{00}^{2}+n_{\pi 0}^{2}+\bar{n}_{\pi 0}^{2}+n_{0 \pi}^{2}+\bar{n}_{0 \pi}^{2}+n_{\pi \pi}^{2}+\bar{n}_{\pi \pi}^{2}}{2} \sum_{m_{1}, m_{2}} \tilde{Z}_{m_{1}} \tilde{Z}_{m_{2}}\right. \\
& +\left(n_{00} n_{\pi \pi}+n_{0 \pi} n_{\pi 0}+\bar{n}_{00} \bar{n}_{\pi \pi}+\bar{n}_{0 \pi} \bar{n}_{\pi 0}\right) \sum_{m_{1}, m_{2}} \tilde{Z}_{m_{1}+1 / 2} \tilde{Z}_{m_{2}+1 / 2} \\
& +\left(n_{00} n_{\pi 0}+\bar{n}_{00} \bar{n}_{\pi 0}+n_{0 \pi} n_{\pi \pi}+\bar{n}_{0 \pi} \bar{n}_{\pi \pi}\right) \sum_{m_{1}, m_{2}} \tilde{Z}_{m_{1}+1 / 2} \tilde{Z}_{m_{2}} \\
& \left.+\left(n_{00} n_{0 \pi}+n_{\pi 0} n_{\pi \pi}+\bar{n}_{00} \bar{n}_{0 \pi}+\bar{n}_{\pi 0} \bar{n}_{\pi \pi}\right) \sum_{m_{1}, m_{2}} \tilde{Z}_{m_{1}} \tilde{Z}_{m_{2}+1 / 2}\right\} \\
& +\left(I_{8}-C_{8}\right)\left\{\left(\bar{n}_{00} n_{00}+\bar{n}_{\pi 0} n_{\pi 0}+\bar{n}_{0 \pi} n_{0 \pi}+\bar{n}_{\pi \pi} n_{\pi \pi}\right) \sum_{m_{1}, m_{2}} \tilde{Z}_{m_{1}} \tilde{Z}_{m_{2}}\right. \\
& +\left(\bar{n}_{00} n_{\pi \pi}+\bar{n}_{0 \pi} n_{\pi 0}+n_{0 \pi} \bar{n}_{\pi 0}+n_{00} \bar{n}_{\pi \pi}\right) \sum_{m_{1}, m_{2}} \tilde{Z}_{m_{1}+1 / 2} \tilde{Z}_{m_{2}+1 / 2} \\
& +\left(n_{00} \bar{n}_{0 \pi}+\bar{n}_{00} n_{0 \pi}+n_{\pi 0} \bar{n}_{\pi \pi}+\bar{n}_{\pi 0} n_{\pi \pi}\right) \sum_{m_{1}, m_{2}} \tilde{Z}_{m_{1}} \tilde{Z}_{m_{2}+1 / 2} \\
& \left.\left.+\left(n_{00} \bar{n}_{\pi 0}+\bar{n}_{00} n_{\pi 0}+\bar{n}_{0 \pi} n_{\pi \pi}+n_{0 \pi} \bar{n}_{\pi \pi}\right) \sum_{m_{1}, m_{2}} \tilde{Z}_{m_{1}+1 / 2} \tilde{Z}_{m_{2}}\right)\right\}
\end{aligned}
$$

The Möbius strip partition function in the transverse channel is then easily found to be:

$$
\begin{aligned}
\tilde{\mathcal{M}}_{I} & =-\frac{\alpha^{\prime}}{R^{2}} \sum_{n_{1}, n_{2}}\left(\alpha_{1} \varepsilon \hat{V}_{8} Z_{2 n_{1}} Z_{2 n_{2}}-\delta_{2} \hat{S}_{8} Z_{2 n_{1}+1} Z_{2 n_{2}}\right) \\
\tilde{\mathcal{M}}_{I I} & =-\frac{\alpha^{\prime}}{R^{2}}\left(\alpha_{1} \varepsilon \hat{V}_{8} Z_{e e}-\beta_{2} \hat{S}_{8} Z_{o o}\right),
\end{aligned}
$$

which becomes in the direct channel:

$$
\begin{aligned}
\mathcal{M}_{I} & =-\frac{1}{2} \sum_{m_{1}, m_{2}}\left(\alpha_{1} \varepsilon \hat{V}_{8}-(-1)^{m_{1}} \delta_{2} \hat{S}_{8}\right) \tilde{Z}_{m_{1}} \tilde{Z}_{m_{2}} \\
\mathcal{M}_{I I} & =-\frac{1}{2} \sum_{m_{1}, m_{2}}\left(\alpha_{1} \varepsilon \hat{V}_{8}-(-1)^{m_{1}+m_{2}} \beta_{2} \hat{S}_{8}\right) \tilde{Z}_{m_{1}} \tilde{Z}_{m_{2}} .
\end{aligned}
$$

The parameter $\varepsilon$ is a sign ambiguity which we discuss below. It is introduced only in the coefficient of $\hat{V}_{8}$ because of the positivity of $\alpha_{1}$ following the parametrization (3.9). As we explain below, it reflects the freedom of 
introducing one of the two types of orientifolds, $\mathrm{O}^{-}$or $\mathrm{O}^{+}$, defined in Table 1.

The integral over the modular parameter $\tau=\tau_{1}+i \tau_{2}$ in the torus amplitude $\mathcal{T}$ is performed over the fundamental domain

$$
\mathcal{F}: \quad-\frac{1}{2} \leq \tau_{1} \leq \frac{1}{2}, \quad \tau_{2} \geq 0, \quad|\tau| \geq 1
$$

The direct (open string) channel amplitudes $\mathcal{K}, \mathcal{A}$ and $\mathcal{M}$ are integrated over $t \in[0, \infty)$. The corresponding expressions in the transverse channel $\tilde{\mathcal{K}}, \tilde{\mathcal{A}}$ and $\tilde{\mathcal{M}}$ are integrated over $l \in[0, \infty)$, obtained by the following transformations:

$$
\begin{array}{rlrlrl}
\tilde{\mathcal{K}} & : & 2 i t & \longrightarrow & \frac{i}{2 t} & \equiv i l \\
\tilde{\mathcal{A}}: & \frac{i t}{2} & \longrightarrow & \frac{2 i}{t} & \equiv i l \\
\tilde{\mathcal{M}} & : & \frac{i t}{2}+\frac{1}{2} & \longrightarrow & \frac{i}{2 t}+\frac{1}{2} \equiv i l+\frac{1}{2} .
\end{array}
$$

The $t$-dependent parameters in the 1.h.s. of the arrows appear as argument of the $\theta$ and $\eta$ functions in the corresponding vacuum amplitudes $\mathcal{K}, \mathcal{A}$ and $\mathcal{M}$, while the $l$-dependent ones on the r.h.s. are the corresponding arguments in $\tilde{\mathcal{K}}, \tilde{\mathcal{A}}$ and $\tilde{\mathcal{M}}$. Finally, the hat on the characters of $\mathcal{M}$ and $\tilde{\mathcal{M}}$ stands as usual for the translation by $1 / 2$ according to eq. (3.12).

The exact particle content of the models is fixed after imposing three consistency conditions: i) the absence of tachyons, ii) the absence of RR tadpoles and iii) the absence of tree-level NS-NS tadpoles. Actually, the last requirement can be satisfied anly for $\varepsilon=+$. However, since in the next section we will relax this condition, we will discuss here both cases with the understanding that NS-NS tadpoles do not vanish for $\varepsilon=-$. The first requirement is satisfied if there are no pairs of brane-anti-brane at short distance (shorter than the string length). This is achieved by taking $n_{y_{1} y_{2}} \bar{n}_{y_{1} y_{2}}=0$, for all $y_{1}, y_{2}$, and by considering the compactification radii transverse to the branes large enough.

The resulting models ${ }^{2}$ are:

- Model A, where all branes (anti-branes) are on top of orientifolds (antiorientifolds). In this case, supersymmetry is not broken locally and the massless open string states present a fermion-boson degeneracy at treelevel and form supersymmetric multiplets. This model can be obtained

\footnotetext{
${ }^{2}$ Here, we restrict our analysis to branes and anti-branes on top of orientifolds. Introduction of Wilson lines would allow to have new models with branes in the bulk.
} 
by:

$$
\begin{array}{cl}
\text { Model A I : } & \bar{n}_{00}=n_{\pi 0}=\bar{n}_{0 \pi}=n_{\pi \pi}=0 \\
& n_{00}+n_{0 \pi}=\bar{n}_{\pi 0}+\bar{n}_{\pi \pi}=16 \\
\text { Model A II }: & \bar{n}_{00}=n_{\pi 0}=n_{0 \pi}=\bar{n}_{\pi \pi}=0 \\
& n_{00}+n_{\pi \pi}=\bar{n}_{\pi 0}+\bar{n}_{0 \pi}=16
\end{array}
$$

- Model B, where all branes (anti-branes) are on top of anti-orientifolds (orientifolds). In this case, supersymmetry is broken locally at the positions of the branes and anti-branes, and the massless open string spectra do not have a fermion-boson degeneracy but satisfy a non-linear supersymmetry 4]. This model corresponds to:

$$
\begin{array}{cl}
\text { Model B I : } & n_{00}=n_{0 \pi}=\bar{n}_{\pi 0}=\bar{n}_{\pi \pi}=0 \\
& n_{\pi 0}+n_{\pi \pi}=\bar{n}_{00}+\bar{n}_{0 \pi}=16 \\
\text { Model B II : } & n_{00}=n_{\pi \pi}=\bar{n}_{\pi 0}=\bar{n}_{0 \pi}=0 \\
& \bar{n}_{00}+\bar{n}_{\pi \pi}=n_{\pi 0}+n_{0 \pi}=16
\end{array}
$$

- Model $\mathrm{C}$ with mixed configuration, where some of the branes are on top of orientifolds and have fermion-boson degenerate massless spectra, while others sit on top of anti-orientifolds and have a non-linearly realized supersymmetry on their worldvolume and non supersymmetric spectrum. We can split these models to two classes: (a) C Ia and C $I I a$, having two non-supersymmetric and two supersymmetric sectors; these can be either on neighboring or on opposite edges of the square of the two compact dimensions (see Fig. 2); (b) C Ib and C IIb, having three or one supersymmetric sectors. Examples of such models are obtained as:

$$
\begin{array}{ll}
\text { Model C I } a, \text { II } a: & n_{00}=n_{\pi 0}=\bar{n}_{0 \pi}=\bar{n}_{\pi \pi}=0 \\
& n_{0 \pi}+n_{\pi \pi}=\bar{n}_{00}+\bar{n}_{\pi 0}=16 \\
\text { Model C Ib, IIb: } & \bar{n}_{00}=n_{\pi 0}=n_{0 \pi}=n_{\pi \pi}=0 \\
& n_{00}=\bar{n}_{\pi 0}+\bar{n}_{0 \pi}+\bar{n}_{\pi \pi}=16
\end{array}
$$

These models can provide a natural setting for further studies of the mediation of supersymmetry breaking in brane models.

A simple inspection of the annulus and Möbius amplitudes in the direct channel shows that, depending the sign ambiguity $\varepsilon= \pm$, the Chan Paton charges of the gauge fields are antisymmetrized or symmetrized, respectively. 

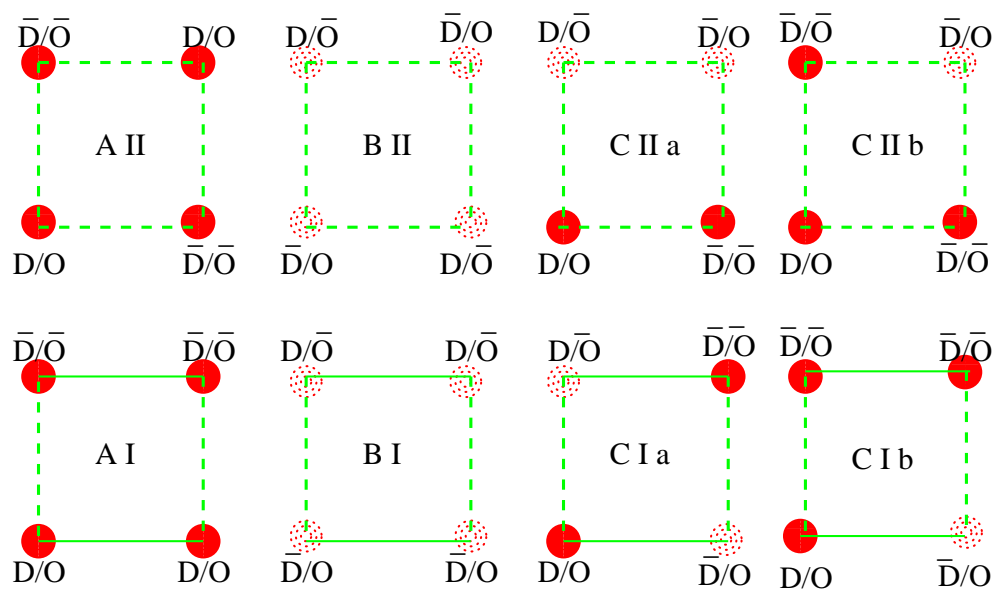

Figure 2: The supersymmetric, non-supersymmetric and mixed models. The dotted lines denote directions along which non-periodic boundary conditions are imposed on the bulk fermions. The plain (dotted) disks stand for locations of supersymmetric (non-supersymmetric) branes.

As a result, in the case $\varepsilon=+$, the gauge group is $\prod_{i} S O\left(n_{i}\right)$ with $n_{i}$ the non-vanishing Chan-Paton factors (here $n_{i}$ denote collectively also $\bar{n}_{i}$ ). In supersymmetric massless sectors, the bosons and fermions belong to vector supermultiplets. In non-supersymmetric massless sectors, the bosons remain in the adjoint (antisymmetric) of $S O(n)$ representation, while fermions are in general in the symmetric ones. In contrast, in the $\varepsilon=-$ case, the gauge group is $\prod_{i} S p\left(n_{i}\right)$ and in non-supersymmetric massless sectors, the bosons are in the adjoint (symmetric) while fermions are in antisymmetric representations.

In the decompactification limit $R \rightarrow \infty$, supersymmetry is restored in the closed string sector while it is broken on the worldvolume of the nonsupersymmetric branes. This is reflected in the non-vanishing of the Möbius amplitude which accounts for the contribution of superstrings stretched between branes and orientifolds and provides the only source for supersymmetry breaking that remains in this limit. As we mentioned before, these models can be used as building blocks for more general constructions in lower dimensions with less supersymmetry, several types of branes and chiral matter representations. 


\section{One-loop effective potential and radius sta- bilization}

\subsection{Explicit examples}

In this section, we compute the one-loop cosmological constant $\Lambda$ in the limit of large transverse radii $R>>l_{s}$. Since the dilaton is considered constant, we will be interested only in the $R$ dependence of the effective potential. The expressions here are given in the string frame.

We first consider the contribution from the torus amplitude (closed string sector) $\Lambda_{c}$. In the limit $R \rightarrow \infty$, the winding modes decouple and we are left over with the zero winding sector:

$$
\begin{aligned}
\Lambda_{c} & \stackrel{R \rightarrow \infty}{\sim} \int_{\mathcal{F}} \frac{d^{2} \tau}{\tau_{2}^{5}} \sum_{m_{1}, m_{2}}\left[Z_{m_{1}, 0} Z_{m_{2}, 0}\left(\left|V_{8}\right|^{2}+\left|S_{8}\right|^{2}\right)-Z_{m_{1}+\frac{1}{2}, 0} Z_{m_{2}+\frac{\epsilon}{2}, 0}\left(V_{8} \bar{S}_{8}+S_{8} \bar{V}_{8}\right)\right] \\
& \stackrel{R \rightarrow \infty}{\sim} \frac{2}{R^{8}} \int_{\mathcal{F}} \frac{d^{2} \tau}{\tau_{2}^{5}} \sum_{m_{1}, m_{2}}\left|\frac{\theta_{2}^{4}}{2 \eta^{12}}\right|^{2}\left(\tau_{1}+i \tau_{2} R\right)\left(e^{-\pi\left(m_{1}^{2}+m_{2}^{2}\right) \tau_{2}}-e^{-\pi\left[\left(m_{1}+\frac{1}{2}\right)^{2}+\left(n_{2}+\frac{\epsilon}{2}\right)^{2}\right] \tau_{2}}\right) \\
& \stackrel{R \rightarrow \infty}{\sim} \frac{2^{7}}{R^{8}} \int_{0}^{\infty} d \tau_{2} \tau_{2}^{4} \sum_{n_{1}, n_{2}}\left(1-(-1)^{n_{1}+\epsilon n_{2}}\right) e^{-\pi \tau_{2}\left(n_{1}^{2}+n_{2}^{2}\right)} \\
& \stackrel{R \rightarrow \infty}{\sim}\left(n_{b}^{c}-n_{f}^{c}\right) \frac{1}{R^{8}}
\end{aligned}
$$

where $\epsilon=0,1$ for the cases $I$ and $I I$, respectively, while in the third line we performed a change of variables $\tau_{2} \rightarrow 1 / \tau_{2}$ and a Poisson resummation in $m_{1}$ and $m_{2}$ to $n_{1}$ and $n_{2}$. Here, $n_{b}^{c}$ and $n_{f}^{c}$ are the number of massless bosons and fermions from the closed string sector. Note that the power of $1 / R$ depends actually on the number of non-compact spacetime dimensions, so that in four-dimensional compactifications the $1 / R^{8}$ becomes $1 / R^{4}$.

We turn now to the contribution $\Lambda_{o}$ of the open (and closed unoriented) string sector, coming from the remaining three surfaces $\mathcal{K}+\mathcal{A}+\mathcal{M}$. Using that the absence of tachyon needs $\alpha_{1}^{2}+\alpha_{2}^{2}-\beta_{1}^{2}-\beta_{2}^{2}+\gamma_{1}^{2}+\gamma_{2}^{2}-\delta_{1}^{2}-\delta_{2}^{2}=0$ 
and that the cancellation of the $\mathrm{RR}$ total charge implies $\beta_{1}=0$, we find:

$$
\begin{aligned}
\left.\Lambda_{o}\right|_{I}= & -\frac{1}{\left(4 \pi^{2} \alpha^{\prime}\right)^{d / 2}} \int_{0}^{\infty} \frac{d l}{l} \\
& {\left[\frac{\theta_{2}^{4}}{\eta^{12}}(i l)\left(\frac{\alpha_{1}^{2}+2^{10}}{2^{8}} \theta_{3}^{2}+\frac{\alpha_{2}^{2}-\beta_{2}^{2}}{2^{8}} \theta_{4}^{2}-\frac{\alpha_{1}^{2}+\alpha_{2}^{2}-\beta_{2}^{2}+2^{10}}{2^{8}} \theta_{3} \theta_{4}\right)\left(\frac{i R^{2}}{2 \alpha^{\prime} l}\right)\right.} \\
& \left.-\frac{\hat{\theta}_{2}^{4}}{\hat{\eta}^{12}}\left(i l+\frac{1}{2}\right)\left(\frac{2^{6}}{2^{8}} \alpha_{1} \varepsilon \theta_{3}^{2}-\frac{2^{6}}{2^{8}} \delta_{2} \theta_{3} \theta_{4}\right)\left(\frac{i R^{2}}{2 \alpha^{\prime} l}\right)\right] \\
\left.\Lambda_{O}\right|_{I I}= & -\frac{1}{\left(4 \pi^{2} \alpha^{\prime}\right)^{d / 2}} \int_{0}^{\infty} \frac{d l}{l} \\
& {\left[\frac{\theta_{2}^{4}}{\eta^{12}}(i l)\left(\frac{\alpha_{1}^{2}+2^{10}}{2^{8}} \theta_{3}^{2}+\frac{\alpha_{2}^{2}-\beta_{2}^{2}-2^{10}}{2^{8}} \theta_{4}^{2}-\frac{\alpha_{1}^{2}+\alpha_{2}^{2}-\beta_{2}^{2}}{2^{8}} \theta_{3} \theta_{4}\right)\left(\frac{i R^{2}}{2 \alpha^{\prime} l}\right)\right.} \\
& \left.-\frac{\hat{\theta}_{2}^{4}}{\hat{\eta}^{12}}\left(i l+\frac{1}{2}\right)\left(\frac{2^{6}}{2^{8}} \alpha_{1} \varepsilon \theta_{3}^{2}-\frac{2^{6}}{2^{8}} \beta_{2} \theta_{4}^{2}\right)\left(\frac{i R^{2}}{2 \alpha^{\prime} l}\right)\right]
\end{aligned}
$$

where $d$ is the number of non-compact dimensions. In the limit $R \rightarrow \infty$, the resulting potential presents a logarithmic divergence. This arises from the integration region $l \rightarrow \infty$ which corresponds to the ultraviolet (UV) limit in the open string channel. In fact, for finite $R$, in the limit $l \rightarrow \infty$, the integrand of the expressions (4.2) and (4.3) is exponentially suppressed as a result of a cancellation between the two $\theta_{3}^{2}$ terms, when $\alpha_{1}=32$ and $\varepsilon=+$ which is the condition for vanishing of the dilaton tadpole.

More precisely, using the properties of $\theta$-functions, one can invert their argument $i R^{2} / 2 \alpha^{\prime} l \rightarrow i 2 \alpha^{\prime} l / R^{2}$ by replacing $\theta_{3} \rightarrow\left(2 \alpha^{\prime} l\right)^{1 / 2} \theta_{3} / R$ and $\theta_{2,4} \rightarrow$ $\left(2 \alpha^{\prime} l\right)^{1 / 2} \theta_{4,2} / R$; one can then take the limit $l \rightarrow \infty$ using their asymptotic expansions, implying $\theta_{3,4}(i l) \rightarrow 1$ and $\theta_{2} \rightarrow 0$ with $\theta_{2} / \eta^{3}(i l) \rightarrow 2$. As a result, one is left over with a quadratic divergence $\sim d l$ proportional to the NS-NS dilaton tadpole coefficient

$$
\frac{2^{3}}{2^{6}} \frac{1}{\left(4 \pi^{2} \alpha^{\prime}\right)^{d / 2}} \int^{\infty} d l: \quad\left(\alpha_{1}^{2}+2^{10}-2^{6} \alpha_{1} \varepsilon\right) \frac{\alpha^{\prime}}{R^{2}}=\frac{\left(\alpha_{1}-2^{5} \varepsilon\right)^{2} \alpha^{\prime}}{R^{2}} .
$$

This divergence can be reproduced in the effective field theory limit. For instance we consider the contribution from one of the $2^{3}$ states present in (4.4) in four dimensions, descring the radion $\varphi$ associated with the two compact dimensions of radius $R$. Note that in section 2 we considered fixed the string coupling and thus constant the ten dimensional dilaton. However, once we turn on fluctuations, it is easy to see that the fields that have orthogonal kinetic terms are the radion and the four-dimensional dilaton. It follows that 
the relevant part of the action which describes the fluctuations of the radion is:

$$
\int d^{4} x\left[\frac{1}{2 \kappa^{2}} \mathcal{R}^{(4)}+(\partial \varphi)^{2}+e^{-\kappa \varphi} \frac{M_{s}^{4}}{g_{s}} V_{0}\right]
$$

where the constant $V_{0}$ is given by the total tension of all branes and orientifolds. For the models we described in section 3.2, it is given by:

$$
V_{0}=\left(\alpha_{1}-2^{5} \varepsilon\right) \frac{\left(\alpha_{1}-2^{5} \varepsilon\right)}{2(2 \pi)^{2}}
$$

Using the action (4.5), one can compute the vacuum diagram that gives rise to the one-loop quadratic divergence (4.4); it is given by the massless on-shell propagator of the radion ending on two tadpoles:

$$
\left.\frac{M_{s}^{8}}{g_{s}^{2}} \frac{\kappa^{2} V_{0}^{2}}{2 p^{2}}\right|_{p^{2}=0}=\frac{1}{2^{5} \pi} \frac{M_{s}^{4}}{(2 \pi)^{4}} \frac{\left(\alpha_{1}-2^{5} \varepsilon\right)^{2}}{R^{2}} \frac{1}{p^{2}}
$$

To compare with the string theory side, we notice that:

$$
\int d l=\left.\int d l q^{\frac{p^{2} \alpha^{\prime}}{4}}\right|_{p^{2}=0}=\left.\frac{2}{\pi \alpha^{\prime} p^{2}}\right|_{p^{2}=0} .
$$

It follows that the field theory result (4.7) reproduces the quadratic divergence of the string expression (4.4) (up to the multiplicative numerical factor $2^{3}$ giving the massless states degeneracy).

On the other hand, taking first the limit $R \rightarrow \infty$, one finds additional contributions from the $\theta_{4}$ terms and one is left over with a logarithmic divergence $d l / l$, as $l \rightarrow \infty$. This phenomenon is related to the fact that tadpoles do not vanish locally (even when they vanish globally for $\alpha_{1}=32$ and $\varepsilon=+$ ), and thus, a new contribution arises in the decompactification limit [13.

A simple inspection shows that the logarithmic divergence comes entirely from the Möbius amplitude, proportional to $\hat{\theta}_{2}^{4} / \hat{\eta}^{12}$ in eqs. (4.2) and (4.3), as expected since it is the only source of supersymmetry breaking in the $R \rightarrow \infty$ limit. This divergence is cut-off by the size of the transverse dimension and leads in four dimensions $(d=4)$ to

$$
\begin{aligned}
\left.\Lambda_{U V}\right|_{I} & \simeq \frac{1}{\left(4 \pi^{2} \alpha^{\prime}\right)^{2}} \int^{\infty} \frac{d l}{l} 2^{4} \times \frac{2^{6}}{2^{8}}\left(\alpha_{1} \varepsilon \theta_{3}^{2}-\delta_{2} \theta_{3} \theta_{4}\right)\left(\frac{i R^{2}}{2 \alpha^{\prime} l}\right) \\
\left.\Lambda_{U V}\right|_{I I} & \simeq \frac{1}{\left(4 \pi^{2} \alpha^{\prime}\right)^{2}} \int^{\infty} \frac{d l}{l} 2^{4} \times \frac{2^{6}}{2^{8}}\left(\alpha_{1} \varepsilon \theta_{3}^{2}-\beta_{2} \theta_{4}^{2}\right)\left(\frac{i R^{2}}{2 \alpha^{\prime} l}\right) .
\end{aligned}
$$


As a result, in the limit $R \rightarrow \infty$, we find a logarithmic behavior:

$$
\Lambda_{U V}(R) \simeq \alpha M_{s}^{4} \ln R
$$

with

$$
\alpha=\frac{8}{\left(4 \pi^{2}\right)^{2}}\left(\alpha_{1} \varepsilon-\delta_{2}\right)=\left.\right|_{\varepsilon=+} \frac{1}{\pi^{4}}\left(n_{\pi \pi}+n_{\pi 0}+\bar{n}_{00}+\bar{n}_{0 \pi}\right)
$$

for model $I$, and

$$
\alpha=\frac{8}{\left(4 \pi^{2}\right)^{2}}\left(\alpha_{1} \varepsilon-\beta_{2}\right)=\left.\right|_{\varepsilon=+} \frac{1}{\pi^{4}}\left(\bar{n}_{\pi \pi}+n_{\pi 0}+\bar{n}_{00}+n_{0 \pi}\right)
$$

for model $I I$. Here, we gave the values only for the case $\varepsilon=+$ which has no tree-level tadpoles (for $\alpha_{1}=32$ ).

Note that the coefficient $\alpha$ of the logarithm is in fact proportional to the difference between the numbers of massless fermions and bosons, in the decompactification limit. The remaining part of the potential leads to a constant $\beta$, plus contributions which vanish in the large radius limit. In order to obtain the effective potential in the Einstein frame, we perform an appropriate rescaling as explained in Section 2, which introduces an overall factor $1 / R^{4}$ :

$$
V_{e f f}^{(1)}(R)=\frac{1}{R^{4}} V_{1}(R) \stackrel{R \rightarrow \infty}{\simeq} \frac{1}{R^{4}}\left(\alpha \ln \left(R M_{s}\right)+\beta\right)
$$

where we used the notation introduced in Section 2.

Unfortunately, it is easy to see that for $\alpha$ positive, the non-trivial extremum of the potential is either not consistent with the approximation $R>>l_{s}$, or is a maximum. Indeed, the extremum $R_{0}$ of $V_{\text {eff }}$ is given by:

$$
R_{0}=l_{s} e^{\frac{1}{4}-\frac{\beta}{\alpha}}
$$

which is in the region $R>>l_{s}$ only for $\beta$ negative. However, since the potential is positive asymprotically, this corresponds to a local maximum, while the minimum corresponds to the run away value $R=\infty$.

Therefore, to find a minimum, we need a model where the coefficient $\alpha$ of the logarithm is negative, implying that there is a surplus of massless bosons versus massless fermions. In fact, as seen from eqs. (4.12) and (4.13), this is the case of the model obtained using the choise $\varepsilon=-$, for which:

$$
\left.\alpha\right|_{\varepsilon=-}=-\frac{1}{\pi^{4}}\left(n_{00}+\bar{n}_{\pi \pi}+\bar{n}_{\pi 0}+n_{0 \pi}\right)
$$


in model $I$, and

$$
\left.\alpha\right|_{\varepsilon=-}=-\frac{1}{\pi^{4}}\left(n_{00}+n_{\pi \pi}+\bar{n}_{\pi 0}+\bar{n}_{0 \pi}\right)
$$

in model $I I$. However, this model has non-vanishing tree-level dilaton tadpole associated to a localized tree-level potential. In the weak coupling limit $g_{s}<1$, this potential dominates over the constant one-loop contribution $\beta$ of eq. (4.14). ${ }^{3}$ It can be easily deduced from the one-loop divergence (4.4), and in the string frame it reads (see eq.(4.6) ):

$$
\frac{1}{g_{s}} V_{0}=\frac{M_{s}^{4}}{8 \pi^{2} g_{s}}\left(32+\alpha_{1}\right)=8 \frac{M_{s}^{4}}{\pi^{2} g_{s}} .
$$

It follows that in this case $V_{\text {eff }}$ has a local minimum at the value:

$$
R_{0}=l_{s} e^{\frac{1}{4}+\frac{8 \pi^{2}}{g_{s}\left(n^{+}+\bar{n}^{+}\right)}}
$$

where $n^{+}\left(\bar{n}^{+}\right)$denotes the total number of D-branes (anti D-branes) located on top of the anti-orientifold $\overline{\mathrm{O}}^{+}$-planes (orientifold $\mathrm{O}^{+}$-planes), given in eqs. (4.16) and (4.17): $n^{+}=n_{00}+n_{0 \pi}, \bar{n}^{+}=\bar{n}_{\pi \pi}+\bar{n}_{\pi 0}$. By taking a value for $g_{s}=g_{Y M}^{2} \simeq .5 \pm .1$, with $g_{Y M}$ the gauge coupling at the string scale, and varying $n^{+}+\bar{n}^{+}$between 1 and 32, it is very easy to obtain hierarchical large values for the ratio $R_{0} / l_{s}$. In Fig. 3 , we plot the prediction for the string scale, as a function of the gauge coupling for three different values of $n^{+}+\bar{n}^{+}=3,4$ and 5 .

\subsection{Generic case}

Here, we generalize the above results to models which have both types of orientifold planes $\mathrm{O}^{+}$and $\mathrm{O}^{-}$(as well as $\overline{\mathrm{O}}^{ \pm}$). Such constructions can be obtained for instance by turning on (quantized) antisymmetric tensor field background. The effective potential receives a tree level contribution given by the total sum of tensions of branes and orientifolds. In the case of orientifold 7-planes (toroidally compactified in four dimensions), it reads:

$$
\frac{1}{g_{s}} V_{0}=\frac{M_{s}^{4}}{8 \pi^{2} g_{s}} Q^{N S}=\frac{M_{s}^{4}}{8 \pi^{2} g_{s}}\left(-8 N^{-}-8 \bar{N}^{-}+8 N^{+}+8 \bar{N}^{+}+n+\bar{n}\right),
$$

\footnotetext{
${ }^{3}$ Note that in the large radius limit, the one-loop quadratic UV divergence (4.4) vanishes, consistently with the absence of tadpole conditions in non-compact space. Of course, strictly speaking, this is true only for more than two bulk dimensions so that the decoupling decompactification limit exists. For $n=2$, one is left over with the logarithmic correction (4.11) that we take into account.
} 


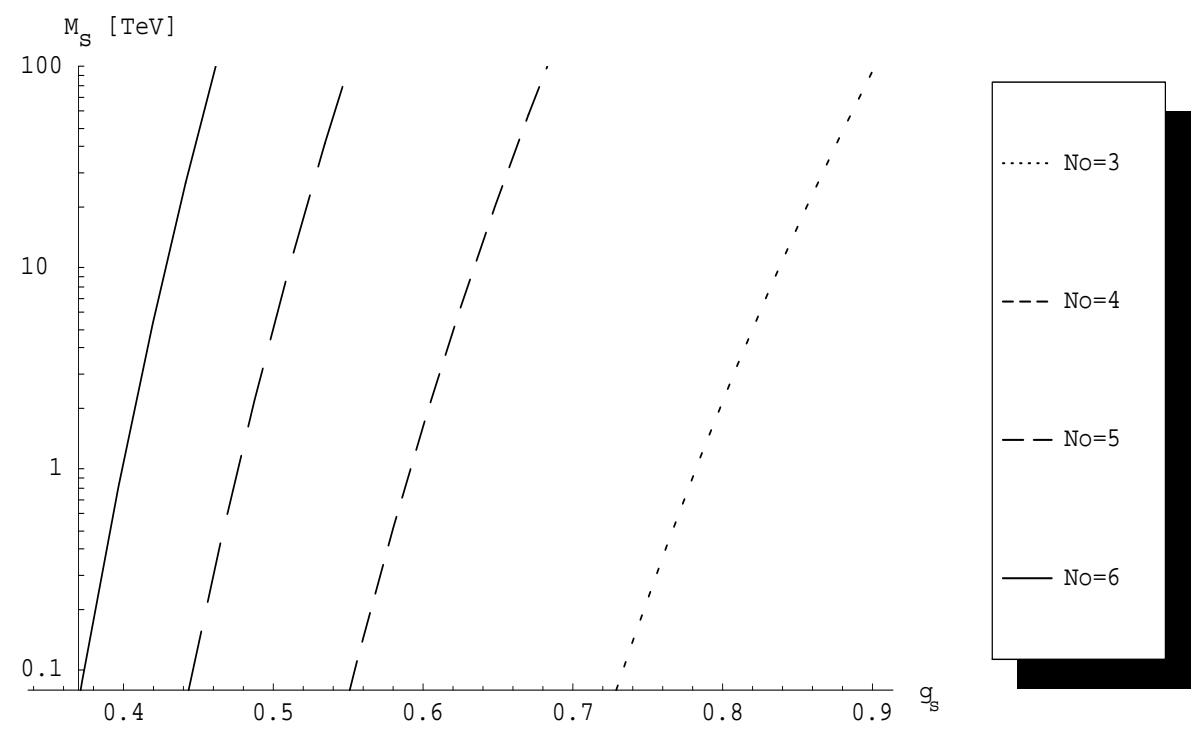

Figure 3: The string scale as a function of the coupling constant for different choices for the number of non-supersymmetric branes.

where $N^{ \pm}\left(\bar{N}^{ \pm}\right)$denote the number of $\mathrm{O}^{ \pm}\left(\overline{\mathrm{O}}^{ \pm}\right)$planes, while $n(\bar{n})$ is the total number of D-branes (anti-branes). On the other hand, in the large transverse radius limit, the one-loop contribution is dominated by the Möbius amplitude, and thus, is proportional to the difference between the numbers of massless fermionic and bosonic degrees of freedom. In the case of two large extra dimensions, it leads to:

$$
\begin{aligned}
V_{1}(R) & \simeq \frac{M_{s}^{4}}{\pi^{4}}\left(\mathcal{N}_{F}-\mathcal{N}_{B}\right) \ln \left(R M_{s}\right) \\
& =\frac{M_{s}^{4}}{\pi^{4}}\left(n^{-}+\bar{n}^{-}-n^{+}-\bar{n}^{+}\right) \ln \left(R M_{s}\right)
\end{aligned}
$$

where $\mathcal{N}_{B}\left(\mathcal{N}_{F}\right)$ is the number of massless bosons (fermions) on the branes, and in analogy with $n^{+}\left(\bar{n}^{+}\right), n^{-}\left(\bar{n}^{-}\right)$denotes the number of branes on anti-orientifolds $\overline{\mathrm{O}}^{-}$(anti-branes on orientifolds $\mathrm{O}^{-}$). Here we neglected the constant term, as it is suppressed by powers of the string coupling $g_{s}$, compared to the tree-level contribution (4.20).

Note that, by inspection of Table 1, the brane configurations appearing in eq. (4.21) are the only ones that break supersymmetry (at the massless level), although, as explained in Section 3, there is still a left-over non-linear supersymmetry. In all other configurations (branes on $\mathrm{O}^{ \pm}$, or anti-branes on $\overline{\mathrm{O}}^{ \pm}$) supersymmetry is linearly realized (at least at the massless level) and there are no logarithmic corrections in the potential. 
It follows that the potential has a minimum at:

$$
R_{0}=e^{\frac{1}{4}+\frac{8 N^{+}+8 \bar{N}^{+}-8 N^{-}-8 \bar{N}^{-}+n+\bar{n}}{n++\bar{n}^{+}-n^{-}-\bar{n}^{-}}} \frac{\pi^{2}}{8 g_{s}} l_{s} .
$$

As in the previous subsection, it is easy to find examples where such a formula gives a size for the compactification radius hierarchically larger than the string length and in the desired range of values.

\section{One-loop contributions to the masses of bulk fields}

\section{$5.1 \quad$ Bulk scalar masses}

Let us consider a generic scalar $\Phi$ living in the closed string bulk. Through its interactions with other particles, it gets a one-loop correction to its mass $g^{2} \Sigma_{\Phi} \Phi^{2}$, where $g$ is the gauge coupling constant, determined by the self energy at zero momentum:

$$
\Sigma_{\Phi}\left(M_{s}, R\right)=\Sigma_{\|}\left(M_{s}, R\right)+\Sigma_{\perp}\left(M_{s}, R\right),
$$

where $\Sigma_{\|}$contains effects exclusively due to other bulk particles, while $\Sigma_{\perp}$ comes from the presence of the boundaries. Here, we restrict our discussion to the case where the generated term quadratic in the field $\Phi$ is just a mass term, while in general there could be also terms localized on the boundaries of the form $\partial_{I} \Phi \partial^{I} \Phi$ or $M \Phi \partial_{I} \Phi$ with $I$ representing one of the transverse directions and $M$ a mass scale.

We first consider the contributions from $\Sigma_{\|}\left(M_{s}, R\right)$. In general, this contribution can be extracted from the torus amplitude. For the case of the radion field in the string models under study, assuming that the full potential has a minimum at a value $R_{0}$, one finds a mass of the order of

$$
\Sigma_{\|} \sim l_{s}^{2+n} / R_{0}^{4+n}
$$

where $n$ is the number of large bulk dimensions with a common radius $R_{0}$. Actually, the same result remains valid for any bulk scalar, which follows simply from the behavior of the torus amplitude as $1 / R_{0}^{2+n / 2}$, for large $R_{0}$.

The contribution $\Sigma_{\perp}$ originates from the other one-loop string surfaces with boundaries and crosscaps. As we have pointed out in section 2, and illustrated by the examples of section 3 , the radion field $\varphi$ acquires a potential given by (in the Einstein frame):

$$
R \rightarrow \infty: \quad V(R) \sim \begin{cases}M_{s}^{4} e^{-4 \kappa \varphi}\left(\alpha \ln \left(R / l_{s}\right)+\beta\right) & \text { for } n=2 \\ M_{s}^{4} e^{-2 n \kappa \varphi}\left(\alpha R^{(2-n)}+\beta\right) & \text { for } n \neq 2\end{cases}
$$


where $\varphi=\kappa^{-1} \ln \left(R / R_{0}\right)$ as defined in section 2 .

For the case of $n=2$, the potential has an extremum at $R_{0}$ with a squared mass $g^{2} \Sigma_{\perp}$ for the radion field $\varphi$, given by:

$$
R_{0}=l_{s} e^{\frac{1}{4}-\frac{\beta}{\alpha}} \quad ; \quad g^{2} \Sigma_{\perp}=-32 \pi \alpha \frac{M_{s}^{4}}{M_{P}^{2}}
$$

so that a minimum at $R_{0}>>l_{s}$ requires $\alpha<0, \beta>0$ and $-\beta / \alpha>>1$.

For $n \neq 2$, the potential in (5.3) gives instead:

$$
R_{0}=l_{s}\left\{-\frac{2 n \beta}{(3 n-2) \alpha}\right\}^{\frac{1}{2-n}} \quad ; \quad g^{2} \Sigma_{\perp}=2 n(2-n) \beta \frac{M_{s}^{4}}{M_{P}^{2}} .
$$

In order to have a minimum at $R_{0}$ with $R_{0}>>l_{s}$ we need $\beta<0$ and $-\beta / \alpha<<1$ for $n>2$ or $\beta>0$ and $-\beta / \alpha>>1$ for $n=1$. Of course, in both cases, unlike the case $n=2$, a large hierarchy cannot be obtained naturally, since there are no exponentials as in (5.4).

The contribution to the mass from $\Sigma_{\perp} \sim M_{s}^{4} / M_{P}^{2} \sim 1 / R_{0}^{n}$ dominates always over the contribution (5.2) from $\Sigma_{\|} \sim 1 / R_{0}^{4+n}$ which can therefore be neglected. Moreover, for $n>2$, the effect of mixing among KK excitations can also be neglected, as the supersymmetry breaking induced mass is suppressed compared to the KK masses by powers of $l_{s} / R_{0}$ for $n>2$, and by a loop factor in the case of $n=2\left(1 / R_{0}^{n / 2}<1 / R_{0}\right)$. Thus, for $n \geq 2$ the radion mass is of the order of $\frac{M_{s}^{2}}{M_{P}}$.

The case of one extra dimension needs a more careful treatment. Because the boundary interactions do not conserve KK momenta, the vacuum polarization diagram associated with $\Sigma_{\perp}$ will connect different mass levels as shown in Fig. 4. We denote by $g(n)$ the coupling of a KK state with momentum $n / R$ with the boundary states ${ }^{4}$ appearing in the one-loop self-energy, and define the tree-level 5D propagator projected on the boundary (at the origin):

$$
\Pi_{5}=\sum_{n} \frac{1}{-p^{2}+\frac{n^{2}}{R^{2}}}
$$

where $p$ is the momentum along the directions parallel to the boundary. We consider now the one-loop correction to the propagator in the large radius limit, $R>>l_{s}$. Since in this limit the series in $\Pi_{5}$ is convergent, we can replace $g(n)=g \sqrt{2-\delta_{n, 0}}$ independent of $n(n \neq 0)$ as long as $n<R l_{s}$. The resulting propagator, obtained by the Dyson resummation shown in Figure

\footnotetext{
${ }^{4}$ In the case of states localized at brane intersections $g(n)=g \sqrt{2-\delta_{n, 0}} \delta^{-n^{2} l_{s}^{2} / R^{2}}$ [14, 4].
} 
4 , is then given by $\left(\Pi_{5}^{-1}-g^{2} \Sigma_{\perp}\right)^{-1}$. Thus, the one-loop corrected mass of $\Phi$ KK excitations can be computed through the equation $\Pi_{5}^{-1}-g^{2} \Sigma_{\perp}=0$ for $p^{2}=M^{2}$.

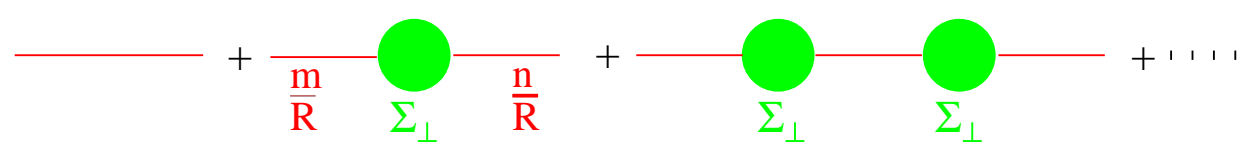

Figure 4: The one-loop self-energy localized on the boundary introduces a mixing between the KK states and modifies the mass spectrum.

The mass eigenstates are then given by the solution of the equation:

$$
\cot (\pi R M)=\frac{\pi R M}{\pi^{2} R^{2} g^{2} \Sigma_{\perp}}
$$

This equation can be solved numerically and leads to a tower of states with masses:

$$
M_{n}=\frac{n+\delta n}{R}, \quad n \geq 0, \delta n<1
$$

where our approximations remain valid for $n<R M_{s}$. The loop of $\Sigma_{\perp}$ leads to a potential for the projection of $\Phi$ on the boundary which modifies the mass of all KK modes by a shift $\delta n$. This shift can be approximated in the limiting cases of very small or very large one-loop self-energy compared to the tree-level masse.

First, in the absence of one-loop self-energy, i.e. $\Sigma_{\perp} \rightarrow 0$, the equation (5.7) gives $\cot (\pi R M)=\infty$ and thus $\delta n \rightarrow 0$. For the scalar fields considered here $R^{2} g^{2} \Sigma_{\perp}>>1$, we can approximate $\cot (\pi R M) \sim \frac{\pi}{2}(1-2 \delta n)$ and we obtain

$$
\delta n \sim \frac{1}{2}-\frac{2 n+1}{2 \pi R^{2} g^{2} \Sigma_{\perp}}
$$

and thus $\delta n \rightarrow 1 / 2$ which is the maximal possible shift. This means that the projection of the field $\Phi$ on the boundary is vanishing in order to minimize the potential $\sim g^{2} \Sigma_{\perp} \Phi^{2}$.

For the radion case in the models under study in this work, we have observed that the main one-loop contribution comes from the Klein, Möbius 
strip and annulus. This is due to open (or closed unoriented) strings and thus gives rise to a boundary potential. Moreover, we have found above that for one dimension $\Sigma_{\perp} \sim 1 / R$, and thus $R^{2} g^{2} \Sigma_{\perp}>>1$, in the large radius limit. This corresponds then to a shift $\delta n \rightarrow 1 / 2$ and all bulk masses of each KK excitations remain of order $1 / R$.

Finally, we would like to comment on the case of a small $\Sigma_{\perp}$ ( which is not realized for the scalar fields discussed here) leading to a small shift $\delta n<<1$ i.e. $R^{2} g^{2} \Sigma_{\perp}<<1$. In this case we can approximate $\cot (\pi R M) \sim 1 /(\pi \delta n)$ which leads to:

$$
(n+\delta n) \delta n \sim R^{2} g^{2} \Sigma_{\perp}
$$

The zero mode mass is then

$$
M_{0}^{2}=\frac{\delta n^{2}}{R^{2}} \sim g^{2} \Sigma_{\perp},
$$

which is the familiar result obtained directly in the effective four-dimensional theory at scales below $1 / R$, while the masses of the other $\mathrm{KK}$ modes are shifted by

$$
\delta n \sim \frac{R^{2} g^{2} \Sigma_{\perp}}{n}
$$

which decreases with $n$.

\subsection{The gravitino mass}

In this subsection, we compute the one-loop correction to the gravitino mass and show that it is of the order of a loop factor times its tree-level mass $1 / 2 R$. The one-loop induced gravitino mass can be read from the two-point correlation function of the corresponding vertex operators. The gravitino vertex in type I string models, present at each boundary can be constructed from the type IIB R-NS and NS-R states by taking the left-right symmetric linear combination.

The one-loop contribution to the gravitino mass can be splitted into two parts, corresponding to the contributions of the $C P$-even and $C P$-odd spin structures. For the computation, we will need the expression of the vertex operators in the $(-1 / 2,0)$ ghost picture:

$$
\begin{aligned}
V_{(-1 / 2,0)}(k, \epsilon) & =\epsilon_{\mu} \int\left[d^{2} z\right]:\left(e^{-\frac{\phi}{2}}(z) u_{\alpha} S^{\alpha}(z)\left(\bar{\partial} X^{\mu}+\alpha^{\prime} k \cdot \tilde{\psi} \tilde{\psi}^{\mu}\right)(\bar{z})\right. \\
& \left.+e^{-\frac{\tilde{\phi}}{2}}(\bar{z})\left(\partial X^{\mu}+\alpha^{\prime} k \cdot \psi \psi^{\mu}\right)(z) \tilde{u}_{\alpha} \tilde{S}^{\alpha}(\bar{z})\right) e^{i k \cdot X}(z, \bar{z}):(5
\end{aligned}
$$


and in the $(-1 / 2,-1)$ ghost picture:

$$
V_{(-1 / 2,-1)}(k, \epsilon)=\epsilon_{\mu} \int\left[d^{2} z\right]:\left(e^{-\frac{\phi}{2}}(z) e^{-\tilde{\phi}}(\bar{z}) u_{\alpha} S^{\alpha}(z) \tilde{\psi}^{\mu}(\bar{z})+c . c .\right) e^{i k \cdot X}(z, \bar{z}):
$$

where $X^{\mu}$ are the (non-compact) bosonic coordinates and $\psi^{\mu}\left(\tilde{\psi}^{\mu}\right)$ their left (right) $2 \mathrm{~d}$ fermionic superpartners. The tilde stands for right-movers and $\phi$ is the super-reparametrization ghost. $S^{\alpha}$ are the ten-dimensional spin fields, $u^{\alpha}$ the associated ten-dimensional spinors and $\epsilon^{\mu}$ the gravitino polarization vector. We will also need the worldsheet supercurrent given by $T_{F}(z)=$ $\psi \partial X(z)+\tilde{\psi} \bar{\partial} X(\bar{z})$.

For the case of even spin structure, we need to compute:

$$
A_{\text {even }}=<V_{(-1 / 2,0)}\left(k, \epsilon^{1}\right)(z, \bar{z}) V_{(-1 / 2,0)}\left(-k, \epsilon^{2}\right)(w, \bar{w}): e^{\phi} T_{F}(v):>\text {. }
$$

All terms in the vertex operators involving powers of the external momentum $k$ are proportional, in the rest frame, to powers of $1 / R$. The only contraction that does not involve explicit powers of $k$ is proportional to:

$$
<\bar{\partial} X^{\mu}(z) \bar{\partial} X^{\nu}(\bar{w}) \partial X^{I}(v) e^{i k \cdot X}(z, \bar{z}) e^{-i k \cdot X}(w, \bar{w})>,
$$

plus permutations of left and right movers. Here, the index $I$ refers either to (non-compact) spacetime or internal indices, collectively. These terms contain three factors of $\partial X$ or $\bar{\partial} X$, and thus, one of them will be contracted with an exponential or replaced by an internal zero mode, leading to a power of $1 / R$.

In the case of odd spin structure, we have to compute instead:

$$
A_{o d d}=<V_{(-1 / 2,-1)}\left(k, \epsilon^{1}\right)(z, \bar{z}) V_{(-1 / 2,0)}\left(-k, \epsilon^{2}\right)(w, \bar{w}) e^{\phi} T_{F}(v) e^{\tilde{\phi}} \tilde{T}_{F}(\bar{v}):>.
$$

A reasoning similar to the above of even spin structure shows that the only contractions that do not involve explicit powers of the external momentum contain three factors of $\partial X$ or $\bar{\partial} X$, which lead again to contributions proportional to $1 / R$.

As a result, the one-loop induced mass term is of order $g_{s} / R$. Note that as for the case of scalar fields, bulk fermions masses might receive one-loop contributions of two kinds: (i) from bulk interactions (ii) from boundary interactions. In the absence of tree level masses, the fermions do not receive one-loop contribution from bulk interactions. This contribution is then proportional to the splitting induced by the non-periodic boundary conditions and vanishes in the decompactification limit. 


\section{Summary}

In conclusion, in this work we studied the brane to bulk mediation supersymmetry in type I string models with brane supersymmetry breaking. Bulk scalar masses are generated at one-loop level and are of order $M_{s}^{2} / M_{P}$, while bulk fermions acquire tree-level masses due to the Scherck-Schwarz boundary conditions and are in general heavier, of the order of the compactification scale $1 / R$. Thus, when the string scale is in the TeV region, the radion acquire a tiny mass and mediates a new attractive universal force at micron distances. We computed its coupling to matter and found that such a force could be detectable in tabletop experiments that test gravity at short distances.

We also studied the particular case of two large bulk dimensions and derived a general formula for the effective potential. We showed that its minimization can stabilize the radion and fix the size of the bulk at values that are hierarchically large than the string length, determining the desired hierarchy between the Planck and string scales. It will be interesting to apply this mechanism in model building of semi-realistic string vacua.

\section{Acknowledgements}

We would like to thank Ann Nelson and Riccardo Rattazzi for valuable comments on an earlier version of the paper. This work was supported in part by the European Commission under RTN contract HPRN-CT-2000-00148, and in part by the INTAS contract N 99-1-590. K.B. acknowledges the financial support provided through the European Community's Human Potential Programme under contract HPRN-CT-2000-00131 Quantum Spacetime. A. L. thanks the Theory Division of CERN for its hospitality and partial financial support.

\section{References}

[1] I. Antoniadis, Phys. Lett. B 246 (1990) 377; I. Antoniadis and K. Benakli, Phys. Lett. B 326 (1994) 69; J. D. Lykken, Phys. Rev. D 54 (1996) 3693.

[2] N. Arkani-Hamed, S. Dimopoulos and G. Dvali, Phys. Lett. B 429 (1998) 263; I. Antoniadis, N. Arkani-Hamed, S. Dimopoulos and G. Dvali, Phys. Lett. B 436 (1998) 257. 
[3] I. Antoniadis, E. Dudas and A. Sagnotti, Phys. Lett. B 464 (1999) 38; G. Aldazabal and A. M. Uranga, JHEP 9910 (1999) 024; G. Aldazabal, L. E. Ibanez and F. Quevedo, JHEP 0001 (2000) 031; C. Angelantonj, I. Antoniadis, G. D'Appollonio, E. Dudas and A. Sagnotti Nucl. Phys. B 572 (2000) 36 .

[4] I. Antoniadis, K. Benakli and A. Laugier, Nucl. Phys. B 631 (2002) 3.

[5] K. Benakli, Phys. Rev. D 60 (1999) 104002; C. P. Burgess, L. E. Ibanez and F. Quevedo, Phys. Lett. B 447 (1999) 257.

[6] E. Dudas and J. Mourad, Phys. Lett. B 514 (2001) 173; G. Pradisi and F. Riccioni, hep-th/0107090.

[7] C. D. Hoyle, U. Schmidt, B. R. Heckel, E. G. Adelberger, J. H. Gundlach, D. J. Kapner and H. E. Swanson, Phys. Rev. Lett. 86 (2001) 1418; J. Chiaverini, S. J. Smullin, A. A. Geraci, D. M. Weld and A. Kapitulnik, hep-ph/0209325, J. C. Long, H. W. Chan, A. B. Churnside, E. A. Gulbis, M. C. Varney and J. C. Price, hep-ph/0210004 D. E. Krause and E. Fischbach, the Casimir Lect. Notes Phys. 562 (2001) 292; H. Abele, S. Haeßler and A. Westphal, in 271th WE-Heraeus-Seminar, Bad Honnef, 25.2.-1.3.2002.

[8] N. Arkani-Hamed, S. Dimopoulos and J. March-Russell, Phys. Rev. D 63 (2001) 064020.

[9] I. Antoniadis and C. Bachas, Phys. Lett. B 450 (1999) 83.

[10] M. Borunda, M. Serone and M. Trapletti, Nucl. Phys. B 653 (2003) 85.

[11] L. Perivolaropoulos and C. Sourdis, Phys. Rev. D 66 (2002) 084018.

[12] S. R. Coleman and E. Weinberg, Phys. Rev. D 7 (1973) 1888; E. Cremmer, S. Ferrara, C. Kounnas and D. V. Nanopoulos, Phys. Lett. B 133 (1983) 61; J. R. Ellis, A. B. Lahanas, D. V. Nanopoulos and K. Tamvakis, Phys. Lett. B 134 (1984) 429; J. R. Ellis, C. Kounnas and D. V. Nanopoulos, Nucl. Phys. B 247 (1984) 373; C. Kounnas, F. Zwirner and I. Pavel, Phys. Lett. B 335 (1994) 403.

[13] J. Polchinski and E. Witten, Nucl. Phys. B 460 (1996) 525 I. Antoniadis, E. Dudas and A. Sagnotti, Nucl. Phys. B 544 (1999) 469.

[14] S. Hamidi and C. Vafa, Nucl. Phys. B 279 (1987) 465; L. J. Dixon, D. Friedan, E. J. Martinec and S. H. Shenker, Nucl. Phys. B 282 (1987) 13. 